\title{
Fission yield measurements at IGISOL
}

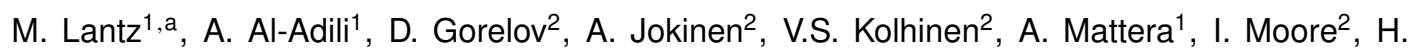 \\ Penttilä $^{2}$, S. Pomp ${ }^{1}$, A.V. Prokofiev ${ }^{1}$, V. Rakopoulos ${ }^{1}$, S. Rinta-Antila ${ }^{2}$, V. Simutkin ${ }^{1,2}$, A. Solders ${ }^{1}$, \\ and the IGISOL group ${ }^{2}$ \\ ${ }^{1}$ Division of Applied Nuclear Physics, Uppsala University, Sweden \\ ${ }^{2}$ Department of Physics, University of Jyväskylä, Finland
}

\begin{abstract}
The fission product yields are an important characteristic of the fission process. In fundamental physics, knowledge of the yield distributions is needed to better understand the fission process. For nuclear energy applications good knowledge of neutroninduced fission-product yields is important for the safe and efficient operation of nuclear power plants. With the Ion Guide Isotope Separator On-Line (IGISOL) technique, products of nuclear reactions are stopped in a buffer gas and then extracted and separated by mass. Thanks to the high resolving power of the JYFLTRAP Penning trap, at University of Jyväskylä, fission products can be isobarically separated, making it possible to measure relative independent fission yields. In some cases it is even possible to resolve isomeric states from the ground state, permitting measurements of isomeric yield ratios. So far the reactions $\mathrm{U}(\mathrm{p}, \mathrm{f})$ and $\mathrm{Th}(\mathrm{p}, \mathrm{f})$ have been studied using the IGISOL-JYFLTRAP facility. Recently, a neutron converter target has been developed utilizing the $\mathrm{Be}(\mathrm{p}, \mathrm{xn})$ reaction. We here present the IGISOL-technique for fission yield measurements and some of the results from the measurements on proton induced fission. We also present the development of the neutron converter target, the characterization of the neutron field and the first tests with neutron-induced fission.
\end{abstract}

\section{Introduction}

The IGISOL facility at University of Jyväskylä has for several decades been used for experiments with exotic nuclei far from the valley of $\beta$ stability. A useful method for producing these highly unstable nuclei is through particle-induced fission of heavy nuclei.

Besides performing experiments on the resulting fission products and their decay products, there are a number of reasons to study the yields of the fission process itself. For a more structured planning of experiments with neutron-rich nuclei, it is essential to have better knowledge of the production cross sections of those nuclei that can be obtained through particle-induced fission.

From a theoretical point of view, accurate knowledge of fission yields is of importance for a better understanding of the fission process. Fission yields also have an impact on the result of the astrophysical rapid neutron capture process, which is responsible for the formation of a large fraction of the nuclei heavier than iron [1]. During the r-process the region of fissionable nuclei is reached, which terminates the r-process path and generates fission products. These have an impact on the resulting

\footnotetext{
ae-mail: Mattias.Lantz@physics.uu.se
} 
stable isotope distribution. The fissioning nuclei are so far from stability that their fission yields have to be calculated based on theoretical models. To improve these models, accurate experimental data on the fission of reachable nuclei, such as ${ }^{238} \mathrm{U}$, is needed.

For nuclear energy applications good knowledge of neutron induced fission product yields is crucial in many aspects, including criticality and reactivity calculations for reactor design, dosimetry and fission gas production for reactor safety and improved burn-up predictions. Good knowledge of the composition of the spent fuel is required for the management of the nuclear waste, i.e. depositories, reprocessing, transmutation and so on. It is also needed for Generation IV reactor concepts, including fast reactors where fast neutrons change the fission yield distribution compared to thermal neutrons. The inventory of fission products in a reactor determines the decay heat, both residual heat after reactor shut down and the decay heat from spent fuel outside of the reactor. Typically, the decay heat is dominated by fission products for the first 50-80 years after extraction of spent nuclear fuel from a reactor. The successful operation of nuclear power plants shows that the current knowledge of the underlying nuclear physics processes is generally sufficient. Predictions of macroscopic reactor parameters with model codes, as well as calculations of the isotopic composition of spent nuclear fuel, are in reasonable agreement with reality. Nevertheless, different evaluations display significant discrepancies for some fission products of relevance [2]. Thus more accurate nuclear data would improve the predictions of fuel compositions and hence both safety and fuel economy.

In view of these needs the objective of the present project is to measure fission yields of high accuracy for different systems, in particular

- the energy dependence of neutron-induced fission yields by varying the incident neutron field,

- the fission yield dependence on the initial system by measuring on different fissile nuclei, and with different incident particles (neutrons, protons, deuterons, ...), and

- other observables such as isomeric yield ratios.

The project is a collaboration between the IGISOL group at University of Jyväskylä and the applied nuclear physics division at Uppsala University.

\section{Experimental method of IGISOL}

IGISOL is an acronym for Ion Guide Isotope Separator On-Line and is a technique that has been developed in Jyväskylä since the early 1980's [3]. The facility has been extensively explained elsewhere [4-8] but some key features will be repeated here. Figure 1 gives a principle view of the steps being described below, part a) of Fig. 4 may also be useful.

The IGISOL technique is based on stopping the nuclear reaction products recoiling out of the reaction target in a helium gas. Here the reaction of interest is fission and thus the reaction products are fission products. The helium gas flows out of the reaction chamber and transports the reaction products towards an electrostatic field that accelerates them through a SextuPole Ion Guide (SPIG). After the SPIG follows further accelerating steps in vacuum, sending the reaction products for analysis or for further treatment.

The reaction products are ionised as they are formed in the nuclear reaction and the high ionisation potential of helium causes a majority of the extracted reaction products to end up as singly charged ions. This significantly simplifies the subsequent mass analysis, where the ions are separated based on their charge to mass $(\mathrm{q} / \mathrm{m})$ ratios by sending them through a dipole magnet.

In earlier versions of IGISOL the nuclides in the isobaric chain selected by the dipole magnet would be identified through $\gamma$-spectroscopy. The introduction of JYFLTRAP, a Penning trap with 7 Tesla magnetic field strength, has significantly improved the experimental method. Before the trap 


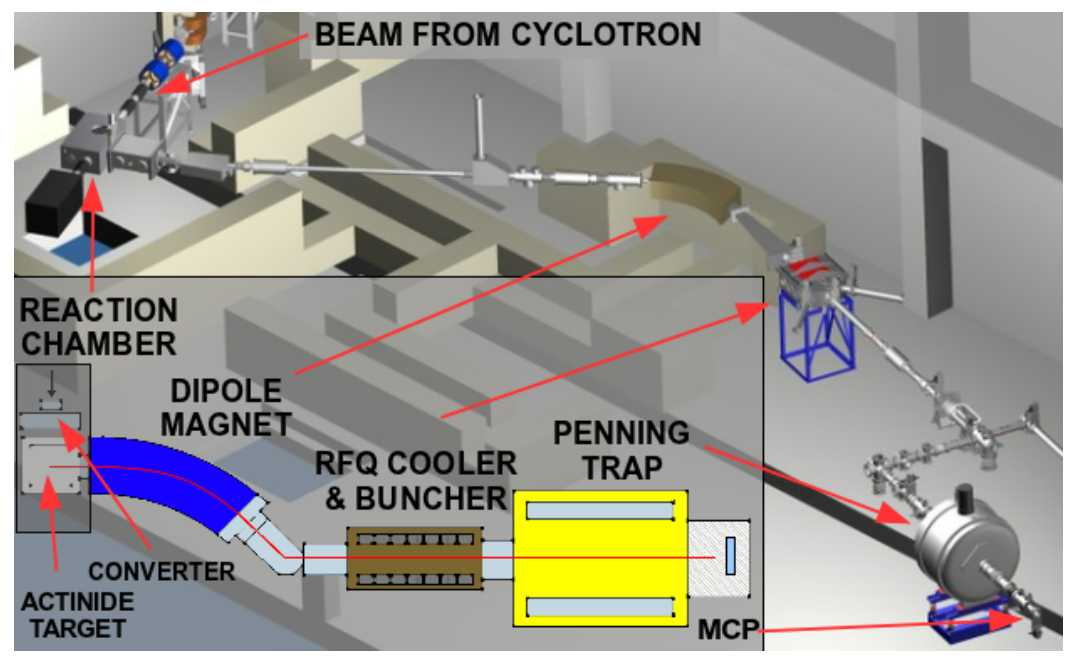

Figure 1. Layout of the IGISOL-JYFLTRAP facility (schematic view in the insert). A particle beam enters from the top left impinging either directly on a fissile target, or alternatively on a neutron converter resulting in a neutron field causing fission in the target. The fission products are accelerated and transported through a dipole magnet for mass selection, followed by an RFQ cooler and buncher for improvement of the beam properties. The cooled ions are injected into the Penning trap for high precision mass separation and finally the count rate is determined by sending the ions to a MCP detector. The acronyms are explained in the text. CAD drawing by Tommi Eronen.

the ions are sent to a radio-frequency quadrupole (RFQ) cooler and buncher, from which they are sent as a bunch to the Penning trap.

The Penning trap can be used as a high-power mass-resolving filter to allow unambiguous identification of the nuclei based on their atomic mass, through the relation between cyclotron frequency, $f_{c}$, and mass of the nuclide, $m$,

$$
f_{c}=\frac{1}{2 \pi} \frac{q}{m} B
$$

where $q$ is the ion charge $(+1)$ and $B$ is the magnetic field strength of JYFLTRAP. The filtering, usually referred as to mass purification, can be performed in less than $100 \mathrm{~ms}$, however, a longer purification time is required for the highest mass resolving power. In the experiments performed so far the total purification time has typically been 0.6-1.3 s. The achieved mass resolution after the Penning trap is of the order of $\frac{A}{\Delta A}=10^{5}$. In some cases it is possible to separate isomeric states from the ground state down to about $200 \mathrm{keV}$ [9].

For the most neutron rich fission products it is necessary to know their half-lives to be able to correct for the losses due to radioactive decay. In addition to the atomic masses and the half-lives no further information about the properties of the fission products are needed, since they are observed by ion counting with multichannel plate (MCP) detectors after JYFLTRAP. It may also be noted that since the detection of the fission products does not require their decay, the yield of very long-lived or even stable fission products is determined equally fast and effectively as that of radioactive nuclides.

The frequency of the Penning trap is scanned to generate a high resolution mass spectrum. One isotope of each element is selected as a reference, against which the yields of the other isotopes are determined. In the measurements of independent fission yields, reference mass spectra are collected 


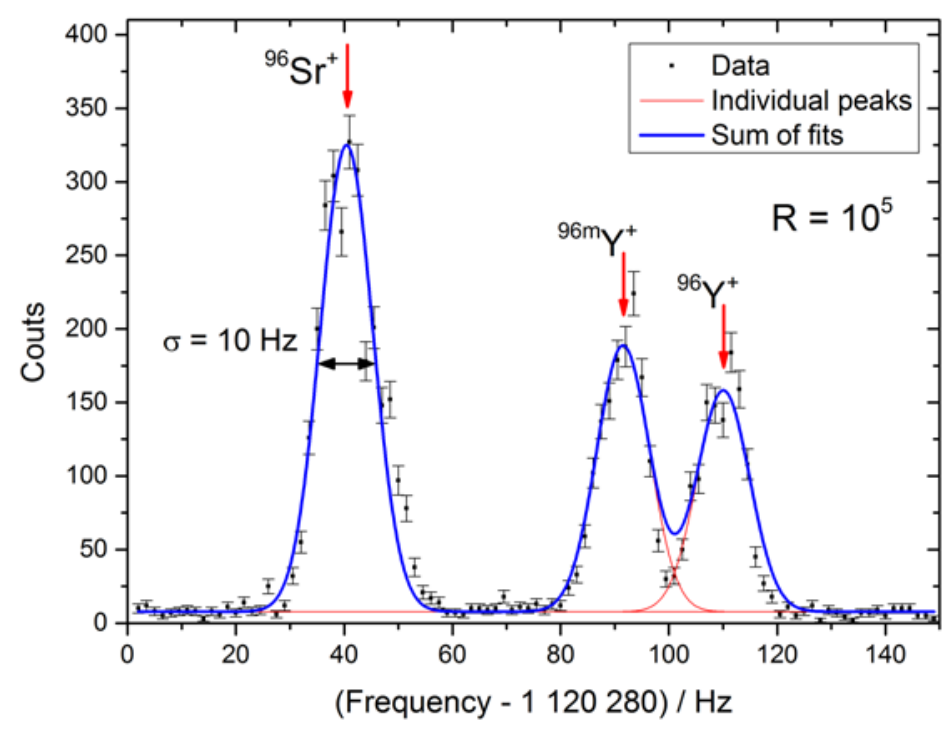

Figure 2. The quadrupole frequency spectrum collected after the Penning trap, showing three identified nuclides of mass 96. Black dots with error bars are the number of counts at each measured frequency. The red lines are gaussian fits to each individual peak (for the ${ }^{96} \mathrm{Sr}$ peak the red line is superimposed by the sum of the fits) and the blue line is the sum of the fits. The isomer ${ }^{96 m} \mathrm{Y}$ at $1.14 \mathrm{MeV}$ excitation energy is easily distinguished from the ground state.

before and after each measurement of the studied isotope. Reference runs may take as long as 20 minutes, while the measurement of the studied isotope can last up to two hours for the lowest production rates. The yield may fluctuate over time in which case it has to be corrected for. After determining the intensity ratio between the mass peaks of the reference and the measured isotopes, applying the appropriate decay corrections and, when necessary, correction for the yield fluctuations, one obtains isotopic yield distributions.

\section{Proton and deuteron induced fission yields}

At IGISOL-JYFLTRAP fission yield experiments have been performed with $25 \mathrm{MeV}$ protons on Th [10] and ${ }^{238} \mathrm{U}$ [11], measuring the isobaric chains for more than 20 different elements. There have also been measurements for a number of nuclei with $50 \mathrm{MeV}$ protons on ${ }^{238} \mathrm{U}$ and with 25 $\mathrm{MeV}$ deuterons on ${ }^{238} \mathrm{U}[4,5]$. The IGISOL method has been developed over time, gradually improving the experimental method and analysis [12]. Comparisons with older data obtained through $\gamma$-spectroscopy give good agreement and show the advantages of the method.

Figure 2 shows an example of the experimental resolution of the method, where the isomer ${ }^{96 m} \mathrm{Y}$ at $1.14 \mathrm{MeV}$ excitation energy can be clearly distinguished from the ground state. 


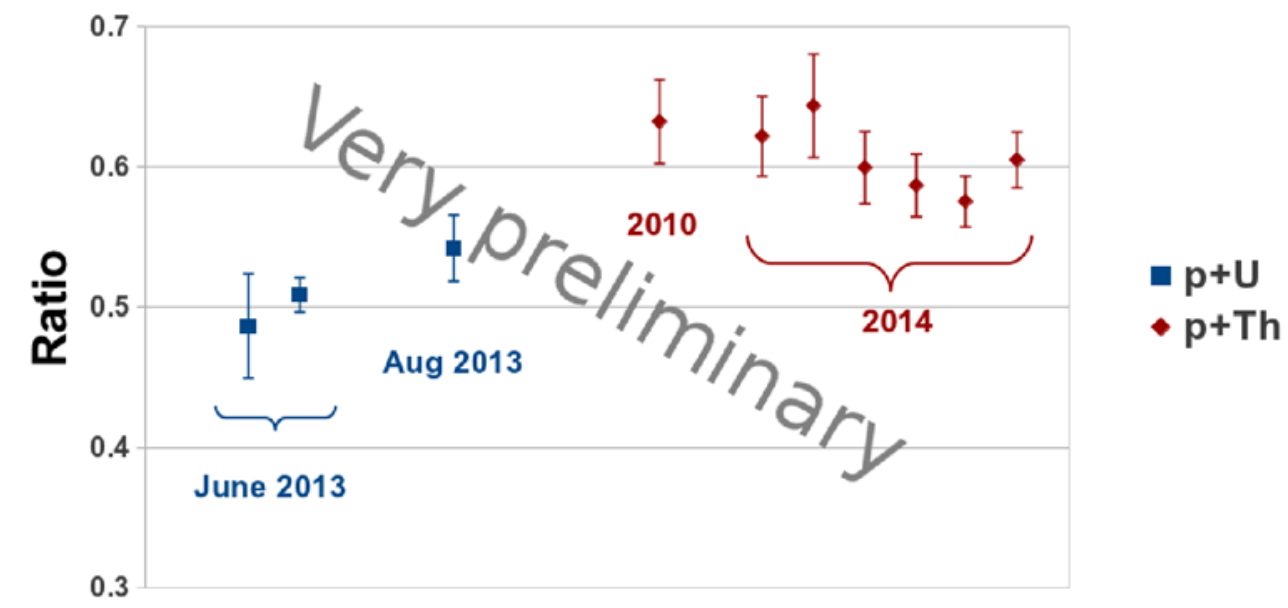

Figure 3. Comparison of isomeric yield ratios for ${ }^{96} \mathrm{Y}$ from $\mathrm{p}+{ }^{238} \mathrm{U}$ (blue) and $\mathrm{p}+\mathrm{Th}$ (red) induced fission from four different experiments. For the June 2013 and 2014 experiments there is more than one measurement of ${ }^{96} \mathrm{Y}$. The error bars only indicate the statistical uncertainty, other corrections have not been implemented yet an may affect both the values and the size of the error bars.

\subsection{Isomeric yield ratios}

One interesting observable to measure is isomeric yield ratios. As there is no need for absolute normalization they are relatively easy to measure, provided that the isomer has long enough half life and an excitation energy large enough to be distinguished with the mass resolution given by JYFLTRAP. Data on isomeric yield radios provide valuable information for theoretical development. Here the isomeric yield ratio is defined as

$$
\text { Ratio }=\frac{\operatorname{Yield}\left({ }^{96 m} Y\right)}{\operatorname{Yield}\left({ }^{96 m} Y\right)+\operatorname{Yield}\left({ }^{96} Y\right)} .
$$

A variable to consider is whether or not the isomeric yield ratios have a dependence on the initial system, i.e. will the ratio be the same for isomers produced from fission of different actinides, or from fission on a given actinide induced by different projectiles? The comprehensive evaluation and compilation by England and Rider [13] assumes no dependence on the initial system and we are unaware of any published experimental results indicating such an effect. From a series of IGISOL measurements with $25 \mathrm{MeV} \mathrm{p}+\mathrm{Th}$ and $25 \mathrm{MeV} \mathrm{p}+{ }^{238} \mathrm{U}$ performed between 2010 and 2014 there are several isomeric pairs to compare [14].

Figure 3 shows preliminary results from the analysis from four different experiments of isomeric yield ratios for the ground state of ${ }^{96} \mathrm{Y}$ and its isomer. The results seem to indicate such a dependence on some isomeric pairs, though all corrections have not been implemented yet to the dataset, and the error bars are likely to increase [15]. For one of the experiments there are also data obtained through $\gamma$-spectroscopy, which will be compared with the results from JYFLTRAP. Since the 2010 experiment the IGISOL-JYFLTRAP facility has been upgraded and moved within the laboratory, so the 2010 data are essentially taken at a different experimental setup. 


\section{a) Proton-induced fission}

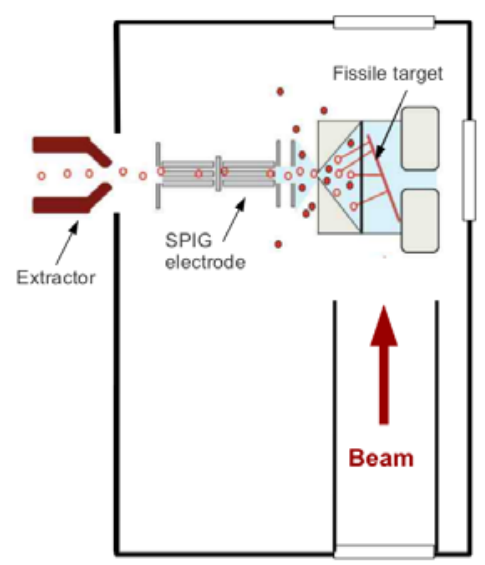

b) Neutron-induced fission

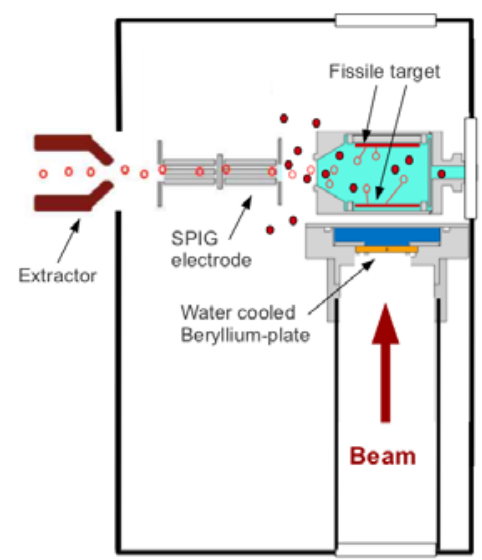

Figure 4. Comparison of the IGISOL chamber for a) experiments with proton/deuteron-induced fission, and b) with a neutron converter for neutron-induced fission. The reaction chamber has a helium flow passing through from the right to left, transporting stopped ions towards the SPIG electrode and an electrostatic field that accelerates the ions towards the RFQ cooler (see Fig. 1). The position of the proton-neutron converter is adjustable and can be mounted further upstream in order to allow the insertion of a moderator for measurements with thermal neutrons.

Future experiments should include further measurements of isomeric yield ratios with proton induced fission. It will also be of interest to measure the same isomeric yield ratios with neutron induced fission.

\section{Neutron induced fission yields}

A project was initiated in 2010 to design a proton-neutron converter in order to measure neutron induced fission yields. Besides the need for nuclear data in view of nuclear energy applications, neutron induced fission enables fundamental research on even more neutron rich nuclei. When the IGISOL-JYFLTRAP facility was moved to a new location it was also supplied with a high intensity MCC30/15 cyclotron [16], which would make it feasible to obtain a high enough neutron flux for measurements of relevance. The ambition was to have a design that allows $10^{12}$ fast neutrons on a large fissile target [17].

After studying different materials and geometries the decided approach was to use a $5 \mathrm{~mm}$ thick Beryllium (Be) plate, slightly thinner than the stopping range for $30 \mathrm{MeV}$ protons. The back side of the plate is cooled by water, and as the majority of the protons stop in the water the cooling requirements are easier to fulfil with a large safety margin [18]. Part b) of Fig. 4 shows the proton-neutron converter assembly with the water cooled Be plate, followed by a cylindrical ion chamber that enables a large area of fissile material, e.g. ${ }^{238} \mathrm{U}$, to be mounted close to the neutron source. The close proximity of the converter allows the ambition for $10^{12}$ fast neutrons to be fulfilled.

A characterisation measurement of the neutron flux from a prototype of the Be converter was performed at the TSL facility in Uppsala by means of two different measurement techniques, time- 


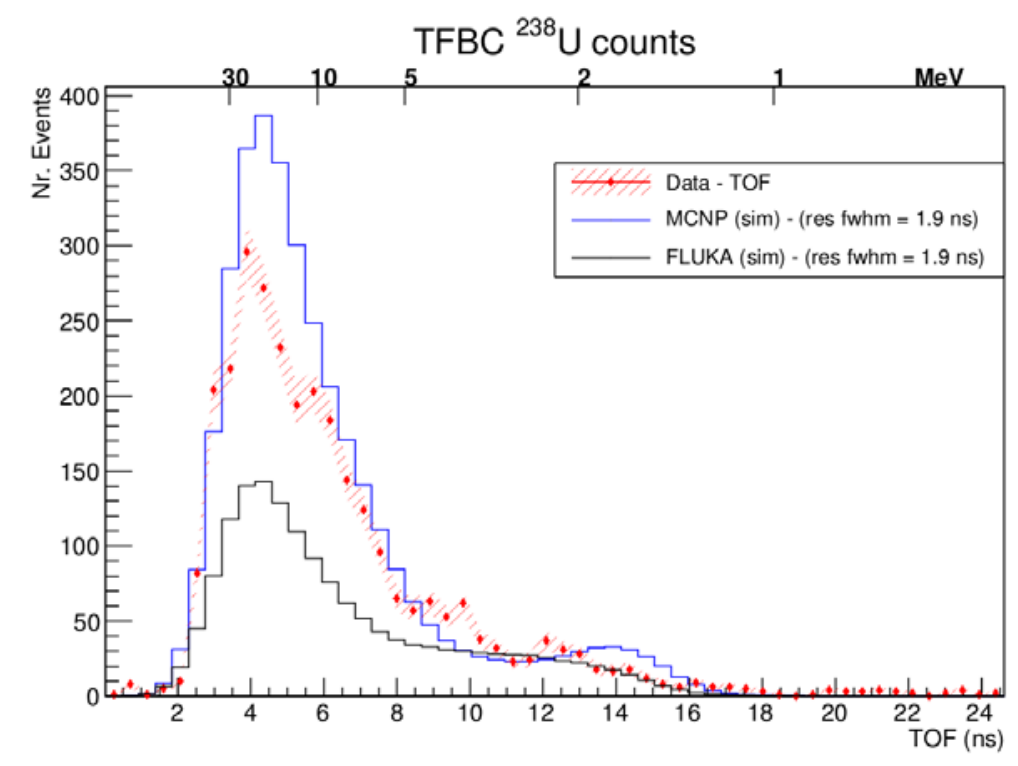

Figure 5. Neutron spectra obtained through ToF with TFBCs at IGISOL. Comparisons with simulations performed with the Monte Carlo codes MCNP and FLUKA.

of-flight measurement (ToF) and Bonner sphere spectroscopy [19-22]. In 2014 the first tests with a prototype at IGISOL were performed. Measurements of the neutron flux within the IGISOL reaction chamber has also been performed [23] with Thin Film Breakdown Counter detectors (TFBCs) [24] and the first attempts to extract ions out of the chamber were performed in 2015 [10].

Simulations of the expected neutron fluxes have been done using the Monte Carlo codes MCNPX [25] and FLUKA [26]. Figure 5 shows a comparison of simulated ToF neutron spectra with experimental data obtained with TFBCs within the IGISOL chamber. The MCNPX simulations agree somewhat better with the experimental values. The discrepancy in absolute magnitude between the two codes is a bit surprising and requires further investigations.

\section{Status and outlook}

The IGISOL-JYFLTRAP facility is very promising for measurements of independent fission yields to complement experimental data from other experimental techniques. Proton and deuteron induced fission yields may be further explored. For nuclear energy applications the possibility to use different neutron fields will be very important.

Initial test runs have been performed with the neutron converter and we foresee actual data taking runs during 2016. In order to fully utilize the new MCC30/15 cyclotron a number of projects have been initialized, for instance

- simulations in order to verify that the neutron field resulting from proton beams with intensity of about $100 \mu \mathrm{A}$ will not cause any issues with respect to radiation protection,

- further characterizations of the neutron fields in order to plan measurements and assess the experimental uncertainties, and 
- modeling and simulation of ion guide geometries and the related physics aspects, in order to optimize and better characterize the yields extracted from the IGISOL reaction chamber [27].

A challenge, but also a possibility, is the relatively long time between the fission event and the actual identification of the fission products. The fastest possible measurement is about $300 \mathrm{~ms}$, though around 1 second is more realistic when accounting for the RFQ cooler and buncher, and the purification time in JYFLTRAP. In comparison with $\gamma$-spectroscopy this is very fast, but it is long when considering that during that time there may have been a number of decays and even beta delayed neutron emission. It is therefore fair to ask what it is that is actually being measured. As some of the upstream decay branching ratios are not known when measuring isomeric pairs, it will be difficult to perform relevant corrections in order to claim a certain isomeric yield ratio. In order to make the data useful for theoretical development it is therefore very important to explain what has been measured and at what time after the fission event. This limitation in the experimental method may also be an opportunity. The time can be increased, which gives a possibility to vary the time for some isomeric pairs with short half lives, and thereby obtain more information about the upstream branching ratios.

The dual purpose of using IGISOL-JYFLTRAP to measure nuclear data for fundamental physics research and simultaneously for applications for neutron induced fission yields lead to some constraints, but also many possibilities. The combination of both purposes strengthen the motivation for the existance of the facility, and as a long-term commitment we foresee many new challenges and valuable nuclear data.

\section{Acknowledgements}

Part of this work has been financed by the Swedish Radiation Safety Authority (SSM), the Swedish Nuclear Fuel and Waste Management Company (SKB), and experimental work through the EU funded research framework programmes "European Research Infrastructures for Nuclear Data Applications" (ERINDA) and "solving CHAllenges in Nuclear DAta" (CHANDA). We also want to acknowledge support from the Academy of Finland under the Finnish Center of Excellence Programme 2012-2017 (Nuclear and Accelerator Based Physics Programme at JYFL) and the project 139382 (Precision fission studies for practical needs).

\section{References}

[1] I.V. Panov, et al., Nucl. Phys. A 747, 633-654 (2005)

[2] B. Lourdel, Research on fission yields evaluation and measurements within the ALFONS project (MSc thesis, Chalmers university of technology and Uppsala university, Göteborg, 2010)

[3] J. Äystö, Nucl. Phys. A 693, 477-494 (2001)

[4] H. Penttilä et al., Eur. Phys. J. A 44, 147-168 (2010)

[5] H. Penttilä et al., Eur. Phys. J. A 48, 43 (2012)

[6] V.S. Kolhinen et al., Nucl. Instrum. Meth. B 317, 506-509 (2013)

[7] H. Penttilä et al., Nuclear Data Sheets 119, 334-337 (2014)

[8] A. Solders et al., Nuclear Data Sheets 119, 338-341 (2014)

[9] K. Peräjärvi et al., Appl. Radiat. Isotopes 68, 450-453 (2010)

[10] D. Gorelov, Nuclear Fission Studies with the IGISOL Method and JYFLTRAP (PhD thesis, University of Jyväskylä, Jyväskylä, 2015)

[11] H. Penttilä et al., manuscript

[12] P. Karvonen, Fission yield studies with SPIG-equipped IGISOL: A novel method for nuclear data measurements (PhD thesis, University of Jyväskylä, Jyväskylä, 2010) 
[13] T.R. England and B.F Rider, Fission product yields per 100 fissions for thermal neutron induced fission decay (Report LA-UR-94-3106, ENDF-349, Los Alamos National Lab, Los Alamos, 1993)

[14] D. Gorelov et al., Acta Physica Polonica B 45, 211-216 (2014)

[15] V. Rakopoulos et al., manuscript

[16] P. Heikkinen, Proc. 18th International Conference On Cyclotrons And Their Applications, 128 (2007)

[17] M. Lantz et al., Phys. Scr. T150, 014020 (2012)

[18] M. Lantz et al., Compendium of Neutron Beam Facilities for High Precision Nuclear Data Measurements, (IAEA-TEKDOC-1743, IAEA, Vienna, 2014) sec 3.2

[19] A. Mattera et al., Nuclear Data Sheets 119, 416-418 (2014)

[20] R. Bedogni et al., manuscript

[21] V. Rakopoulos et al., EPJ Web of Conferences 66, 11032 (2014)

[22] A. Mattera Characterization of a Neutron Source for Fission Yield Studies (PhLic thesis, Uppsala university, Uppsala, 2014)

[23] B. Eriksson, Neutron field characterization using TFBCs and comparison to Monte Carlo simulations (BSc thesis, Uppsala university, Uppsala, 2015)

[24] A.N. Smirnov et al., Nucl. Instrum. Meth. A 687, 14-22 (2012)

[25] G.W. McKinney et al., MCNPX 2.7.0 - New Features Demonstrated, (Report LA-UR-12-25775, Los Alamos National Lab, Los Alamos, 2012)

[26] T.T. Böhlen et al., Nuclear Data Sheets 120, 211-214 (2014), A. Ferrari et al., FLUKA: a multiparticle transport code (Report CERN-2005-10 (2005), INFN/TC_05/11, SLAC-R-773, CERN, Geneva, 2005)

[27] A. Al-Adili et al., Eur. Phys. J. A 51, 59 (2015) 